\title{
Transmission of light through quantum heterostructures modulated by coherent acoustic phonons
}

\author{
Gia-Wei Chern, Kung-Hsuan Lin, and Chi-Kuang Sun ${ }^{\text {a) }}$ \\ Graduate Institute of Electro-Optical Engineering and Department of Electrical Engineering, \\ National Taiwan University, Taipei 10617, Taiwan, Republic of China
}

(Received 4 August 2003; accepted 10 November 2003)

\begin{abstract}
We investigate the optical transmission oscillation of multiple quantum wells (MQWs) under the modulation of coherent acoustic phonons. We treat the coherent acoustic phonons as a semiclassical wave obeying continuum elastic equations. Starting from the microscopic electron-phonon interaction Hamiltonian, we obtain expressions for optical absorption modulation of the MQWs due to coherent acoustic phonons. The acoustic phonons introduce a renormalization to single-particle energy and furthermore modify the resonant condition of photon absorption. The optical transmission modulation can be conveniently expressed with the use of sensitivity functions. We derive the analytical expressions for the sensitivity functions by which we calculate the optical transient transmission changes. The calculated results are in good agreement with the experimental observations. (C) 2004 American Institute of Physics. [DOI: 10.1063/1.1637957]
\end{abstract}

\section{INTRODUCTION}

Coherent acoustic phonons (CAPs) have attracted considerable attention in the past decade due to their potential applications in nano-sized solid-state devices. The inexorable interactions between electrons and phonons provide us with a tool to transport and control carriers using coherent acoustic waves. For example, carrier transport induced by gigahertz surface acoustic waves (SAWs) has been demonstrated in GaAs quantum wires. ${ }^{1,2}$ In addition, acoustically driven storage of light in a quantum well has also been proposed and demonstrated by transforming light into elementary excitations confined in piezoelectric potentials created by the moving SAW. ${ }^{3}$

The rapid advances in ultrafast science and nanotechnology have enabled us to generate and control large-amplitude CAPs. ${ }^{4-13}$ Terahertz (THz) CAPs have been generated using ultrashort laser pulses in bulk solids, ${ }^{4-7}$ semiconductor superlattices,${ }^{8,9}$ quantum dots, ${ }^{10}$ metallic nanoparticles,${ }^{11}$ and a number of other materials. Recently, we reported large coherent LA-phonon oscillations in piezoelectric $\mathrm{InGaN} / \mathrm{GaN}$ multiple quantum wells (MQWs). ${ }^{12,13}$ Coherent control within a few oscillation cycles was also demonstrated in this system. $^{14-16}$

On the theoretical side, Sanders et al. recently proposed a microscopic theory based on generalized density-matrix formalism for the generation of coherent LA phonons in InGaN/GaN MQW. ${ }^{17}$ A time-dependent Hartree-Fock approach, including many-body Coulomb interaction, was used to describe the ultrafast photogeneration of electrons and holes within the QW. The authors in Ref. 17 showed that, under appropriate conditions, the photogeneration of coherent LA phonons can be mapped onto a loaded string model. Recently, we also made a comparison between the loadedstring model and the experimental results by studying the

\footnotetext{
${ }^{a)}$ Electronic mail: sun@cc.ee.ntu.edu.tw
}

dependence of phonon spectra on the well-to-barrier thickness ratio of a MQW. An overall agreement was obtained. ${ }^{16}$

The photogenerated CAPs have a high degree of temporal coherence, similar to photons in a laser. As a result, we may treat these CAPs as macroscopic elastic waves in the semiclassical approximation. Through both piezoelectric and deformation potential couplings, these coherent elastic waves modify the particle self-energies and introduce couplings between carriers among different subbands of the MQW. Consequently, the optical absorption of the MQW is also modulated by these acoustic phonons. By measuring the probe transmission at different time delays, we can observe the time dynamics of CAPs in InGaN MQWs. Please note that the absorption changes due to piezoelectric coupling is the same as the quantum-confined Franz-Keldysh effect. ${ }^{18,19}$ In addition, we shall show that this effect will dominate the absorption modulation compared with the deformation potential coupling.

The effects of CAPs on MQW absorption can be conveniently described using a sensitivity function. ${ }^{20}$ The probe transmission change can be expressed as an overlapping integral of the sensitivity function with the time-dependent strain field. We shall derive expressions for the MQW sensitivity function in the following section. It has two contributions: the piezoelectric and deformation potential couplings. In addition, it is found that the piezoelectric coupling is the dominating factor over the deformation potential coupling.

The paper is organized as follows. In Sec. II, we present a continuum theory for photogeneration of CAPs in InGaN/ GaN MQWs. Section III is about the phonon-induced optical absorption modulation of a MQW. We shall treat CAPs as a semiclassical wave and study the effects on the confined carriers. The CAP phonon modifies the interband electron-hole density matrix elements and furthermore modulates the sample absorption. We derive expressions for the differential transmission. A sensitivity function will be introduced in Sec. IV. Using the sensitivity function, the time-resolved dif- 
ferential transmission can be calculated. We also compare it with the experimental result. Finally, we draw conclusions in Sec. V.

\section{GENERATION OF COHERENT LA PHONONS}

In this section, we shall present a continuum theory for the generation of coherent LA phonons in InGaN/GaN MQWs. The generation mechanism can be understood as follows. ${ }^{13,17}$ The photoexcited electrons and holes are spatially separated by the large piezoelectric field within the wells. This separation results in a periodic space-charge density within the MQW. The quasiperiodic space-charge density partially screens the piezoelectric field and impulsively changes the mechanical equilibrium state to an inhomogeneous one through piezoelectric and deformation potential couplings. As a result, the lattice relaxes to this new inhomogeneous equilibrium and thereby initiates the coherent acoustic oscillations.

A microscopic theory based on generalized density matrix method has been proposed ${ }^{17}$ to explain the photoexcitation of carriers and the subsequent generation of coherent phonons in InGaN MQW's. Under typical experimental conditions, the authors showed that the microscopic theory could be simplified and mapped onto a loaded-string problem that can be easily solved and understood. This loaded-string equation can also be obtained based on piezoelectric constitutive relations, continuum elastic mechanics, and Poisson equations. ${ }^{21}$ We shall present the continuum theory in this section. Here, we consider only generation of coherent LA phonons in a (0001)-grown InGaN MQW. The propagation direction of a LA phonon is along the $c$ axis, which is also the $z$ axis in our coordinate system.

The constitutive equations for a piezoelectric semiconductor are

$$
\begin{aligned}
& T_{i}=C_{i j} S_{j}-e_{k i} E_{k}+\delta_{i} \sum_{\nu=e, h} d_{\nu} \rho_{\nu}, \\
& D_{i}=\varepsilon_{i} E_{i}+e_{i j} S_{j} .
\end{aligned}
$$

Here, we use the reduced index convention for symmetric indices. Also note that repeated indices imply summation. In these expressions, $T_{i}$ is the stress tensor, $S_{i}$ is the strain tensor. $C_{i j}, \varepsilon_{i}, e_{k i}$ are the stiffness, dielectric, and piezoelectric constants of the semiconductor. The stress also has a contribution from deformation potential coupling, which is represented by the last two terms of $T_{i} . d_{e}$ and $d_{h}$ are the deformation potential constants, and $\rho_{e}, \rho_{h}$ are carrier densities of electron and hole, respectively. Here, we introduce $\delta_{i}=1$ for $i=1,2,3$, and $\delta_{i}=0$ for $i=4,5,6$. This delta function means that the deformation potential coupling contributes only to the diagonal parts of the stress tensor.

For a (0001)-grown InGaN MQW, only the LA phonon propagating along the $z$ axis will be photoexcited. We denote $u$ as the $z$ component of the displacement vector, which is the only nonvanishing component of the displacement. The system has a cylindrical symmetry about the $c$ axis. As a result, all quantities depend only on $z$. The governing equations for the time evolution of MQW system are

$$
\rho_{0} \frac{\partial^{2} u}{\partial t^{2}}=\frac{\partial T_{3}}{\partial z}
$$

$$
\frac{\partial D_{3}}{\partial z}=\rho_{\mathrm{sc}}=|e|\left[\rho_{h}(z, t)-\rho_{e}(z, t)\right],
$$

$$
\frac{\partial \rho_{\nu}}{\partial t}=\beta_{\nu} I(t)
$$

where $\rho_{0}$ is the mass density, $|e|$ is the fundamental electric charge, $\rho_{h}$ and $\rho_{e}$ are the number density of hole and electron, respectively, and $I(t)$ is the normalized pump-pulse intensity. Equation (3) describes the motion of the displacement $u$, and Eq. (4) is the Poisson equation for electric displacement $D_{i}$. The space charge $\rho_{\mathrm{sc}}$ serves as the driving source for the system. We use a simple rate Eq. (5) to model the photogeneration process. Here $\beta_{\nu}$ is the phenomenological absorption constant for carrier species $\nu$. In Ref. 17, the authors used generalized density matrix method to calculate the photogeneration of electrons and holes. The many-body Coulomb interaction was treated in the screened dynamic Hartree-Fock approximation. From their calculation, the many-body effect will decrease the total photogenerated carrier density for equal pump intensity. This is due to the dynamic quantum-confined Stark effect. However, the generation curve of the photocarriers is well described using the simple rate equation.

Due to the small difference between $\operatorname{In}_{0.1} \mathrm{Ga}_{0.9} \mathrm{~N}$ and $\mathrm{GaN}$, we regard the MQW as a homogeneous material. By substituting the constitutive Eq. (2) into Eq. (4), we obtain the expression for electric field

$$
E_{3}=\frac{1}{\varepsilon_{3}} \int_{-\infty}^{z} d z^{\prime} \rho_{\mathrm{sc}}\left(z^{\prime}\right)-\frac{e_{33}}{\varepsilon_{3}} S_{3} .
$$

The electric field is related to the strain through piezoelectric coupling. By substituting Eq. (6) and Eq. (1) into Eq. (3), we have the following loaded-string equation for $u$ :

$$
\begin{aligned}
\rho_{0} \frac{\partial^{2} u}{\partial t^{2}}-\hat{C} \frac{\partial^{2} u}{\partial z^{2}} & =f_{\text {piezo }}+f_{\text {def }} \\
& =-\frac{e_{33}}{\varepsilon_{3}} \rho_{\mathrm{sc}}+\left[d_{e} \frac{\partial \rho_{e}}{\partial z}+d_{h} \frac{\partial \rho_{h}}{\partial z}\right],
\end{aligned}
$$

where $\hat{C}=C_{33}+e_{33}^{2} / \varepsilon_{3}$ is the effective stiffness constant for the LA phonon propagating along $c$ axis. We introduce two driving forces: $f_{\text {piezo }}$ and $f_{\text {def }}$. The first one comes from the piezoelectric coupling. This force is proportional to the space-charge density $\rho_{\mathrm{sc}}$ and the piezoelectric constant $e_{33}$. The second force is due to deformation potential coupling. It is proportional to the spatial derivatives of the carrier density.

The loaded-string equation can be solved using Green's function method, as discussed in Ref. 17. Here, we solved the loaded-string equation for the case of an InGaN/GaN MQW with four wells. The In composition is 0.1 and the well and barrier thicknesses are 22 and $70 \AA$, respectively. We use the $\mathbf{k} \cdot \mathbf{p}$ method ${ }^{18}$ to calculate the electron and hole wave functions in a $22-\AA / 70-\AA \operatorname{In}_{0.1} \mathrm{Ga}_{0.9} \mathrm{~N} / \mathrm{GaN}$ MQWs. The MQW is assumed to be embedded in a $p-i-n$ structure $^{17}$ 


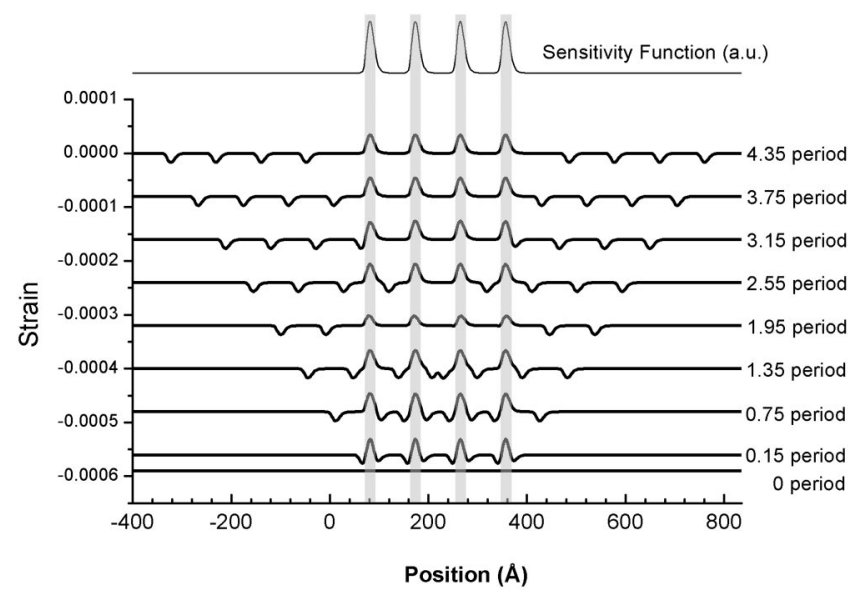

FIG. 1. Calculated strain fields at consecutive time delays (with spacing equal to $0.6 T$ ). Also shown is the calculated sensitivity function for MQW with four QWs.

with an applied electric field. The magnitude of the applied field is adjusted to produce a periodic band diagram of the MQW. The parameters used in the calculation can be found in Ref. 17.

In Fig. 1, we show the time evolution (traces from bottom to top with increasing time) of the strain field of coherent LA phonons. The results shown in the figure correspond to strain distribution at consecutive time delays with $0.6 T$ spacing, where $T$ is the fundamental oscillation period. As can be seen from the figure, a static carrier-induced strain exists in the MQW after two counterpropagating acoustic wave trains leave the MQW region. Also shown in the figure is the calculated sensitivity function. The transient probe transmission change is the overlapping integral of the sensitivity function with the strain fields at different time delays. We will discuss more about the sensitivity function in the following section.

\section{ACOUSTICALLY MODULATED OPTICAL TRANSMISSION}

We now consider the influences of CAPs on the quantum-confined carriers in the MQW. It is found that the existence of CAPs introduces a renormalization to the singleparticle energy and causes couplings between subbands of the same carrier type. Here, we mainly follow the formalism developed by Sanders et al. in Ref. 17. By solving the photogeneration of CAPs using the simplified macroscopic approach in Sec. II, we use the density matrix formalism outlined in Ref. 17 to explain how this propagating coherent wave modulates the optical absorption of carriers. Explicitly, the microscopic polarization is obtained by solving the interband density matrix equation under the assumption that the electrons adiabatically follow the motion of coherent phonons. The relaxation time scales for carriers to reach a quasi-equilibrium in nitride-based materials are usually less than a picosecond. Compared with the oscillation period of coherent acoustic phonons, which is about tens of picoseconds, this adiabatic assumption is reasonable. By treating the phonon-induced energy renormalization as a perturbation, we obtain analytic expressions for the optical transmission changes.

The interaction between electrons and LA phonons in a MQW can be described using the following microscopic Hamiltonian: ${ }^{17}$

$$
\mathcal{H}_{e A}=\sum_{\alpha, n, n^{\prime}, \mathbf{k}} \int \frac{d q}{2 \pi} \mathcal{M}_{n, n^{\prime}}^{\alpha}(k, q) D(q, t) c_{\alpha, n, \mathbf{k}}^{\dagger} c_{\alpha, n^{\prime}, \mathbf{k}},
$$

where $c_{\alpha, n, \mathbf{k}}^{\dagger}$ and $c_{\alpha, n, \mathbf{k}}$ are the electron creation and annihilation operators, respectively. $\alpha$ represents carrier species, (i.e., electrons or holes), $n, n^{\prime}$ are the subband indices, and $\mathbf{k}$ is the in-plane wave vector. ${ }^{17}$ Here, only LA phonons propagating along the $c$ axis of the MQW are considered. $D(q, t)$ is the coherent phonon amplitude with wave vector $\mathbf{q}=q \hat{\mathbf{z}}$. This amplitude is related to the Fourier transform of strain as $D(q, t)=\widetilde{S}_{3}(q, t) / q$.

The Hamiltonian in Eq. (8) describes the scattering of electron from subband state $\left|\alpha, n^{\prime}, \mathbf{k}\right\rangle$ to subband state $|\alpha, n, \mathbf{k}\rangle$. The transition matrix element includes deformation potential coupling and piezoelectric coupling: ${ }^{17}$

$$
\begin{aligned}
\mathcal{M}_{n, n^{\prime}}^{\alpha}(k, q)= & \sqrt{\frac{\hbar^{2}}{2 \rho_{0}\left(\hbar \omega_{q}\right) V}} \\
& \times\left[i q \mathcal{D}_{n, n^{\prime}}^{\alpha}(k, q)-\frac{|e| e_{33}}{\epsilon_{3}} \mathcal{P}_{n, n^{\prime}}^{\alpha}(k, q)\right],
\end{aligned}
$$

where $V$ is the crystal volume, $\omega_{q}=c_{s} q$ is the phonon oscillation frequency, and $c_{s}=\sqrt{\hat{C} / \rho_{0}}$ is the sound velocity along [0001] direction. In Eq. (9), $\mathcal{D}_{n, n^{\prime}}^{\alpha}(k, q)$ and $\mathcal{P}_{n, n^{\prime}}^{\alpha}(k, q)$ are form factors responsible for the deformation potential and piezoelectric couplings, respectively. A detailed calculation and discussion of these matrix elements can be found in Ref. 17. The explicit expression of these factors will be listed in the next section, when we calculate the sensitivity function.

It is noted that in Eq. (8), we regard the phonon amplitude $D(q, t)$ as a classical quantity, as is the case for the electric field $\mathbf{E}(t)$ of a laser. The dynamics of $D(q, t)$ is governed by the classical equation of motion described in Sec. II. This is the analogy of the semiclassical treatment of laser-atom interaction. In such a treatment, the quantum fluctuations are neglected. It is plausible in our case due to the high coherence of the phonon modes under consideration.

The quasi-cw optical absorption constant of the MQW can be obtained by solving the equation of motion for the interband electron density matrix, or the effective Bloch equation, ${ }^{22,23}$ under the assumption that carriers have reached a quasi-equilibrium. Since our derivation in this section mainly follows the formalism of Ref. 17, we shall maintain the notations used in this reference. The electron density matrix is defined as ${ }^{17} N_{n, n^{\prime}}^{\alpha, \alpha^{\prime}}(\mathbf{k}, t)=\left\langle c_{\alpha, n, \mathbf{k}}^{\dagger}(t) c_{\alpha^{\prime}, n^{\prime}, \mathbf{k}}(t)\right\rangle$, where $\langle\ldots\rangle$ denotes the statistical average of the quasi-equilibrium state of the system. The intraband components of the density matrix $N_{n, n^{\prime}}^{\alpha, \alpha}(\mathbf{k}, t)$ describe correlations between different subbands of the same carrier species and are related to the generation of CAPs. If $n=n^{\prime}, N_{n, n}^{\alpha, \alpha}(\mathbf{k}, t)=f_{n}^{\alpha}(\mathbf{k}, t)$ is just the 
quasi-equilibrium carrier distribution for carrier $\alpha$ in subband $n$. Here, we consider the solution of the interband components $N_{n, n^{\prime}}^{c, v}(\mathbf{k}, t)$ and $N_{n^{\prime}, n}^{v, c}(\mathbf{k}, t)$, which represent coherence between conduction and valence electrons in subbands $n$ and $n^{\prime}$, respectively. These elements are related to the optical polarization.

In the quasi-cw approximation, the electric field of the input laser is expressed as $\mathbf{E}(t)=\operatorname{Re}\left[\hat{\boldsymbol{\epsilon}} \mathcal{E}(t) e^{i \omega t}\right]$. The polarization can be written similarly as $\mathbf{P}(t)=\operatorname{Re}\left[\hat{\boldsymbol{\epsilon}} \mathcal{P}(t) e^{i \omega t}\right]$. The optical absorption constant $\alpha$ is related to the imaginary part of the susceptibility defined as $\chi=\mathcal{P} / \varepsilon_{0} \mathcal{E}$. Due to the rapid varying exponential factor $e^{i \omega t}$, the slowly varying envelope function for interband polarization is introduced as $N_{n, n^{\prime}}^{c, v}(k, t)=\tilde{N}_{n, n^{\prime}}^{c, v}(k, t) e^{i \omega t}$. Under the rotating wave approximation, the equations of motion for $\tilde{N}_{n, n^{\prime}}^{c, v}(k, t)$ $\operatorname{are}^{17,22,23}$

$$
\begin{aligned}
i \hbar \frac{\partial \widetilde{N}_{n, n^{\prime}}^{c, v}(k, t)}{\partial t}= & -\left\{E_{n}^{c}(k)-E_{n^{\prime}}^{v}(k)-\hbar \omega\right. \\
& \left.+i \hbar \gamma_{n, n^{\prime}}\right\} \widetilde{N}_{n, n^{\prime}}^{c, v}(k)+d_{n^{\prime}, n}^{v, c}(k) \mathcal{E}(t) \\
& \times\left\{f_{n^{\prime}}^{v}(k, t)-f_{n}^{c}(k, t)\right\}-\left\{Q_{n, n}^{c}(k, t)\right. \\
& \left.-Q_{n^{\prime}, n^{\prime}}^{v}(k, t)\right\} \widetilde{N}_{n, n^{\prime}}^{c, v}(k) \\
& -\sum_{m \neq n, n^{\prime}}\left\{Q_{n, m}^{c}(k) \widetilde{N}_{m, n^{\prime}}^{c, v}(k)\right. \\
& \left.-\widetilde{N}_{n, m}^{c, v}(k) Q_{m, n^{\prime}}^{v}(k)\right\} .
\end{aligned}
$$

In this equation, $E_{n}^{c}(k)$ and $E_{n^{\prime}}^{v}(k)$ are the single-particle energies of electrons and holes, respectively, $\gamma_{n, n^{\prime}}=1 / T_{2}$ is the phenomenological dephasing constant, and $d_{n^{\prime}, n}^{v, c}(k)$ are the dipole matrix elements. It is noted that $d_{n^{\prime}, n}^{v, c} \mathcal{E} / \hbar$ are the Rabi frequencies of the incident light. $Q_{n, m}^{\alpha}(k, t) / \hbar$ is the acoustic analogy of Rabi frequency, and the expression for $Q$ is given by ${ }^{17}$

$$
Q_{n, n^{\prime}}^{\alpha}(k, t)=\int \frac{d q}{2 \pi} D(q, t) \mathcal{M}_{n, n^{\prime}}^{\alpha}(k, q) .
$$

One can see the similarities between this expression and the definition of Rabi frequency. However, the effects on the interband polarization are quite different. The first term on the right-hand side of Eq. (10) describes the detuning of oscillation frequencies between electric field and interband polarization. The second term represents the interaction with laser light. A quasi-equilibrium distribution has been assumed for electrons and holes within the subbands. The laser field contributes to the establishment of the interband polarizations. Both the third and fourth terms are due to CAPs. The third term produces an additional frequency detuning to the polarization. Its effects can be incorporated into the definition of a time-dependent, single-particle energy: ${ }^{.7}$

$$
\mathcal{E}_{n}^{\alpha}(k, t)=E_{n}^{\alpha}(k)+Q_{n, n}^{\alpha}(k, t) .
$$

This change in single-particle energies causes a shift of the resonant wave vector, which in turn changes the optical absorption of the MQW. The last term on the right-hand side of
Eq. (10) represents couplings between different interband polarizations caused by CAPs. This term would result in some interesting phenomena when two or more interband transitions are involved by photoexcitation of a laser with broad spectrum.

In the preceding treatment, we have neglected Coulomb corrections in order to obtain an analytic expression for the optical absorption constant. We also assume that the carriercarrier scattering and carrier-LO-phonon scattering quickly drive the electrons and holes to quasi-equilibrium states with effective temperature equal to that of lattice system. In addition, the intraband polarizations are neglected in Eq. (10) due to the fast dephasing time constant.

Here, we consider optical transition involving only one conduction subband and one valence subband; for example, $n=\mathrm{C} 1$ to $n^{\prime}=\mathrm{HH} 1$. Transitions involving multiple subbands will be discussed elsewhere. As mentioned earlier, for such multisubband transitions, in addition to the phonon-induced energy renormalization, there are couplings between subbands of the same carrier type caused by CAPs.

Assuming that carriers adiabatically follow the motion of CAPs, we have $\partial \widetilde{N}_{n, n^{\prime}}^{c, v} / \partial t \simeq 0$. From Eq. (10), we obtain

$$
\begin{aligned}
\widetilde{N}_{n, n^{\prime}}^{c, v}(k, t)= & \frac{1}{2} d_{n^{\prime}, n}^{v, c}(k) \mathcal{E}(t) \\
& \times \frac{\left\{f_{n^{\prime}}^{v}(k, t)-f_{n}^{c}(k, t)\right\}}{\left\{\mathcal{E}_{n}^{c}(k, t)-\mathcal{E}_{n^{\prime}}^{v}(k, t)-\hbar \omega\right\}+i \hbar \gamma_{n, n^{\prime}}} .
\end{aligned}
$$

The distribution functions $f_{n}^{c}(k, t)$ and $f_{n^{\prime}}^{v}(k, t)$ are time dependent due to the influence of phonons. Under the assumption of quick relaxation, both subbands remain in quasiequilibrium with their own Fermi levels:

$$
f_{n}^{\alpha}(k, t)=\frac{1}{\exp \left\{\left[\mathcal{E}_{n}^{\alpha}(k, t)-E_{\mathrm{F}}^{\alpha}(t)\right] / k T\right\}+1},
$$

where $E_{\mathrm{F}}^{\alpha}$ is the quasi-Fermi energy for carrier species $\alpha$. Since the carrier energy $\mathcal{E}_{n}^{\alpha}(k, t)$ is time varying, the quasiFermi energy also changes with time in order that the total number of carriers is conserved. If the phonon-induced renormalization $Q_{n, n}^{\alpha}$ is independent of the wave vector $k$, the time dependence of quasi-Fermi energy cancels that of the single-particle energy, resulting in a time-independent distribution function $f(k)$.

The system polarization is given by $\mathcal{P}=\operatorname{tr}[\hat{d} \hat{N}]$ $=\varepsilon_{0} \chi \mathcal{E}$, where the trace includes summation over the inplane wave vectors. From this, we obtain the susceptibility $\chi$, and the absorption constant is related to its imaginary part as $\alpha(\omega)=\left(\omega / c n_{s}\right) \operatorname{Im} \chi(\omega)$ :

$$
\begin{aligned}
\alpha(\omega)= & -\frac{C_{0}}{V} \sum_{\mathbf{k}}\left|p_{n, n^{\prime}}^{c, v}(k)\right|^{2} \mathcal{I}_{n, n^{\prime}}(k) \\
& \times \frac{\left(\gamma_{n, n^{\prime}} / \pi\right)\left[f_{n^{\prime}}^{v}(k, t)-f_{n}^{c}(k, t)\right]}{\left[\mathcal{E}_{n}^{c}(k, t)-\mathcal{E}_{n^{\prime}}^{v}(k, t)-\hbar \omega\right]^{2}+\left(\hbar \gamma_{n, n^{\prime}}\right)^{2}},
\end{aligned}
$$


where $\mathcal{I}_{n, n^{\prime}}(k)$ is the overlapping integral of electron and hole wave functions, ${ }^{18}$ and the constant $C_{0}$ $=\pi|e|^{2} / \varepsilon_{0} n_{s} c m_{0}^{2} \omega$.

In the limit of $\gamma_{n, n^{\prime}} \rightarrow 0$, the Lorentzian factor becomes a delta function, and the absorption can be simplified as

$$
\begin{aligned}
\alpha(\omega)= & -C_{0} \frac{2}{L_{z}} \int \frac{d k}{2 \pi} k\left|p_{n, n^{\prime}}^{c, v}(k)\right|^{2} \mathcal{I}_{n, n^{\prime}}(k)\left[f_{n^{\prime}}^{v}(k, t)\right. \\
& \left.-f_{n}^{c}(k, t)\right] \delta\left[\mathcal{E}_{n}^{c}(k, t)-\mathcal{E}_{n^{\prime}}^{v}(k, t)-\hbar \omega\right] .
\end{aligned}
$$

To derive this equation, we assume cylindrical symmetry for subband dispersions. The resonant wave vector corresponding to photon energy $\hbar \omega$ is given by the following energyconservation condition:

$$
\hbar \omega=E_{n}^{c}(k)-E_{n^{\prime}}^{v}(k)+Q_{n, n}^{c}(k, t)-Q_{n^{\prime}, n^{\prime}}^{v}(k, t) .
$$

We can convert the integration over $k$ in Eq. (16) to the integration over energy $E$ by introducing the following effective mass:

$$
\frac{1}{m_{n, n^{\prime}}(k)}=\frac{1}{m_{n}^{c}(k)}-\frac{1}{m_{n^{\prime}}^{v}(k)}=\frac{1}{\hbar^{2} k}\left(\frac{\partial E_{n}^{c}}{\partial k}-\frac{\partial E_{n^{\prime}}^{v}}{\partial k}\right) .
$$

With the use of $\delta$ function, we obtain

$$
\alpha(\omega)=\alpha_{0}(k)\left[f_{n^{\prime}}^{v}(k, t)-f_{n}^{c}(k, t)\right],
$$

where the wave vector $k=k(t)$ is determined by Eq. (17). The intrinsic absorption constant $\alpha_{0}$ is

$$
\alpha_{0}(k)=-\frac{m_{n, n^{\prime}}(k)}{m_{0}} \frac{|e|^{2}\left|p_{n, n^{\prime}}^{c, v}(k)\right|^{2}}{L_{z} n_{s} c \varepsilon_{0} \hbar^{2} \omega m_{0}} \mathcal{I}_{n, n^{\prime}}(k) .
$$

We now consider the changes of optical absorption due to coherent LA phonons. The existence of a coherent LA phonon causes renormalization $Q$ to the single-particle energies, and therefore shifts the resonant wave vector [cf. Eq. (17)]. Let $k_{0}$ be the resonant wave vector in the absence of coherent phonon; that is, $Q=0$. It satisfies $\hbar \omega=E_{n}^{c}\left(k_{0}\right)$ $-E_{n^{\prime}}^{v}\left(k_{0}\right)$. We regard $Q$ as a small perturbation, the shift of resonant wave vector $\delta k$ can be expanded to linear order in $Q$ as

$$
\delta k=-\frac{m_{n, n^{\prime}}\left(k_{0}\right)}{\hbar^{2} k_{0}}\left[Q_{n, n}^{c}\left(k_{0}, t\right)-Q_{n^{\prime}, n^{\prime}}^{v}\left(k_{0}, t\right)\right] .
$$

Figure 2 shows a schematic diagram of the acoustically modulated subband dispersions. The energy bands are shifted vertically by phonon-induced renormalization $Q$. We use vertical arrows to represent optical transitions with incoming photon energy $\hbar \omega$. For a fixed photon energy, this acoustic modulation results in a shift $\delta k$ of the resonant in-plane wave vector, which is given by Eq. (21).

There are two contributions to the modulation of the absorption. One is from the $k$-dependent intrinsic absorption $\alpha_{0}(k)$. The shift of wave vector $k$ also changes the effective mass, the dipole moment, and the electron-hole overlapping, as can be seen from Eq. (20). The other is from modulations of the electron and hole distribution functions, also due to the shift in resonant wave vector. Thus, to the first order in $Q$, we have the following absorption change

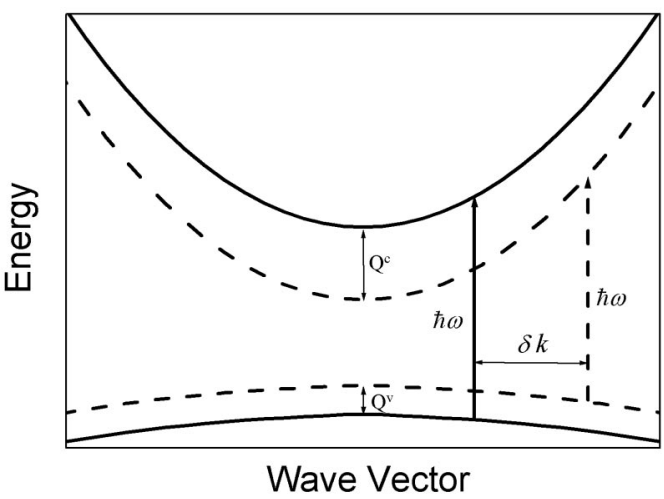

FIG. 2. Schematic diagram of the acoustically modulated subband dispersions. $Q^{c}$ and $Q^{v}$ are the phonon-induced energy renormalizations for electrons and holes, respectively. For a specific input photon energy $\hbar \omega$, this modulation causes a shift $\delta k$ of the resonant wave vector.

$$
\begin{aligned}
\delta \alpha(\omega, t)= & -\frac{m_{n, n^{\prime}}}{\hbar^{2} k_{0}}\left[\frac{\partial \alpha_{0}}{\partial k}\left(f_{n^{\prime}}^{v}-f_{n}^{c}\right)+\alpha_{0}\left(\frac{1}{m_{n^{\prime}}^{v}} \frac{\partial f_{n^{\prime}}^{v}}{\partial E}\right.\right. \\
& \left.\left.-\frac{1}{m_{n}^{c}} \frac{\partial f_{n}^{c}}{\partial E}\right)\right]\left[Q_{n, n}^{c}(t)-Q_{n^{\prime}, n^{\prime}}^{v}(t)\right] .
\end{aligned}
$$

In this expression, quantities depending on $k$ are evaluated at $k=k_{0}$. The differential transmission is given by $\Delta T / T$ $=-\delta \alpha L$. From Eq. (22), the amplitude of absorption change is proportional to the phonon-induced energy renormalization $Q$. It is also proportional to the differential of Fermi functions. The differential $\partial f / \partial E$ has a peak as the resonance is close to the quasi-Fermi level. As a result, the observed differential transmission signal will be enhanced for the same phonon amplitude.

\section{SENSITIVITY FUNCTION}

The transient transmission changes $\Delta T / T$ of probe pulse due to the existence of coherent LA phonons can be conveniently expressed using a sensitivity function ${ }^{20}$

$$
\left(\frac{\Delta T}{T}\right)_{\mathrm{LA}}(t)=\int_{-\infty}^{\infty} d z S_{3}(z, t) \mathcal{F}(z ; \omega) .
$$

For a given strain amplitude $S_{3}$ of a small volume element $A d z$ located at position $z$, the quantity $\mathcal{F}(z)$ represents the weighted contribution of this strain value to the sample transmission change.

The sensitivity function in the classical limit can be easily obtained as follows. Considering a multilayer structure with negligible quantum tunneling. The absorption constant of $i$ th layer is $\alpha_{i}$. For a perturbing strain $S_{3}$, the absorption change is $\delta \alpha_{i}=\left(\partial \alpha_{i} / \partial S_{3}\right) S_{3}$. As a result, the sensitivity function assumes a constant value in each layer, that is, $\mathcal{F}(z)=-\partial \alpha_{i} / \partial S_{3}$ for $z$ lying in the $i$ th layer.

In the case of InGaN MQW, due to quantum tunneling of carriers, the transmission sensitivity of a local point is related to the entire distribution of wave functions within the MQW. In order to obtain the sensitivity function, we first express the phonon-induced self-energy $Q$ by the strain field $S_{3}(z, t)$. 
The phonon amplitude $D(q, t)$ is the Fourier transform of the displacement field $u(z, t)$. Its relation to the strain $S_{3}$ is

$$
D(q, t)=\frac{1}{i q}\left[\frac{\hbar^{2}}{2 \rho_{0}\left(\hbar \omega_{q}\right) V}\right]^{-1 / 2} \int_{-\infty}^{\infty} d z e^{-i q z} S_{3}(z, t) .
$$

Substituting this expression into Eq. (11), we have

$$
Q_{n, n^{\prime}}^{\alpha}(k, t)=\int_{-\infty}^{\infty} d z S_{3}(z, t) \mathcal{G}_{n, n^{\prime}}^{\alpha}(k, z),
$$

where we introduce the following sensitivity function for energy renormalization:

$$
\begin{aligned}
\mathcal{G}_{n, n^{\prime}}^{\alpha}(k, z)= & \int_{-\infty}^{\infty} \frac{d q}{2 \pi} e^{-i q z}\left[\mathcal{D}_{n, n^{\prime}}^{\alpha}(k, q)\right. \\
& \left.-\frac{1}{i q} \frac{|e| e_{33}}{\varepsilon_{3}} \mathcal{P}_{n, n^{\prime}}^{\alpha}(k, q)\right] .
\end{aligned}
$$

These functions are just Fourier transforms of the transition matrix elements in Eq. (9). As has been described in the previous section, relative strengths of the various transitions are determined by form factors $\mathcal{D}_{n, n^{\prime}}^{\alpha}$ and $\mathcal{P}_{n, n^{\prime}}^{\alpha}$. The derivation of these form factors can be found in Ref. 17. The form factor for screened piezoelectric coupling is

$$
\mathcal{P}_{n, n^{\prime}}^{\alpha}(k, q)=\sum_{j} \int_{-\infty}^{\infty} d z e^{i q z} F_{n, k, j}^{\alpha}(z) F_{n^{\prime}, k, j}^{\alpha}(z),
$$

while the form factor for deformation potential coupling is

$$
\mathcal{D}_{n, n^{\prime}}^{\alpha}(k, q)=\sum_{j} \Theta_{j}^{\alpha} \int_{-\infty}^{\infty} d z e^{i q z} F_{n, k, j}^{\alpha}(z) F_{n^{\prime}, k, j}^{\alpha}(z),
$$

where $F_{n, k, j}^{\alpha}(z)$ is the envelope function of the $n$th quantized state for carrier species $\alpha$, and $j$ is the spinor index of the Bloch basis state $|\alpha, j\rangle .{ }^{17}$ The form factors for the two couplings are similar, except that in summing over spinor components $j$, the terms are weighted by $j$-dependent deformation potentials $\Theta_{j}^{\alpha}$, definitions of which can be found in Ref. 17.

Substituting these expressions into Eq. (26), we obtain

$$
\begin{aligned}
\mathcal{G}_{n, n^{\prime}}^{\alpha}(k, z)= & \sum_{j} \Theta_{j}^{\alpha} F_{n, k, j}^{\alpha}(z) F_{n^{\prime}, k, j}^{\alpha}(z) \\
& -\sum_{j} \frac{|e| e_{33}}{\varepsilon_{3}} \int_{z}^{\infty} d z^{\prime} F_{n, k, j}^{\alpha}\left(z^{\prime}\right) F_{n^{\prime}, k, j}^{\alpha}\left(z^{\prime}\right) .
\end{aligned}
$$

The energy renormalization $Q$ is overlapping integral of the strain field $S_{3}(z, t)$ with the sensitivity function $\mathcal{G}_{n, n}^{\alpha}(z)$. There are two contributions to this function. One is from the deformation potential couplings, which is the first term on the right-hand side of Eq. (29). It is proportional to the weighted sum of deformation potential coefficients $\Theta_{j}^{\alpha}$. The second term on right-hand side of Eq. (29) represents the piezoelectric contribution. Note that it is a monotonically increasing function of $z$, because of the existence of a static carrier-induced strain with the MQW. ${ }^{16,17}$ This term is proportional to the piezoelectric constant $e_{33}$, and is effectively equivalent to the well-known quantum-confined FranzKeldysh effect. Just as the piezoelectric coupling dominates

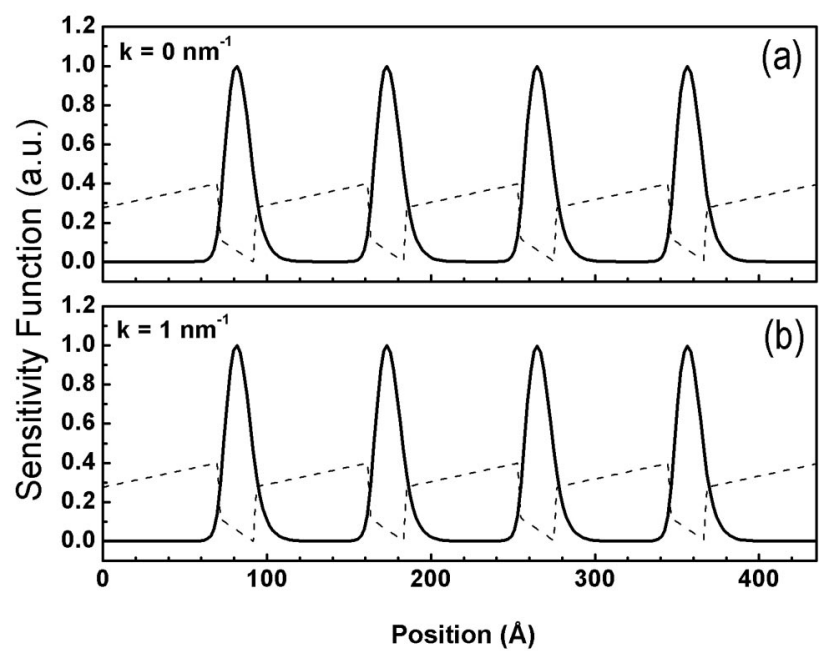

FIG. 3. The normalized sensitivity function related to $\mathrm{HH} 1$ and $\mathrm{C} 1$ at transverse wave vector (a) $k_{t}=0$ and (b) $k_{t}=1 \mathrm{~nm}^{-1}$.

the photogeneration of CAPs. ${ }^{16,17,24}$ In our calculations to be discussed subsequently, we also found that the piezoelectric coupling is also the dominating contribution to the system sensitivity function.

From Eqs. (22) and (25), the overall sensitivity function $\mathcal{F}(z ; \omega)$ is given by

$$
\begin{aligned}
\mathcal{F}(z ; \omega)= & \frac{m_{n, n^{\prime}} L}{\hbar^{2} k_{0}}\left[\frac{\partial \alpha_{0}}{\partial k}\left(f_{n^{\prime}}^{v}-f_{n}^{c}\right)+\alpha_{0}\left(\frac{1}{m_{n^{\prime}}^{v}} \frac{\partial f_{n^{\prime}}^{v}}{\partial E}\right.\right. \\
& \left.\left.-\frac{1}{m_{n}^{c}} \frac{\partial f_{n}^{c}}{\partial E}\right)\right]\left[\mathcal{G}_{n, n}^{c}(z)-\mathcal{G}_{n^{\prime}, n^{\prime}}^{v}(z)\right] .
\end{aligned}
$$

In Figs. 3 and 4, we show the calculated $z$ dependence of sensitivity functions for a four-well $\mathrm{In}_{0.1} \mathrm{Ga}_{0.9} \mathrm{~N} / \mathrm{GaN}$ MQW. From Eq. (30), the $z$ dependence of $\mathcal{F}(z)$ is determined by the difference of the self-energy functions $\mathcal{G}_{n, n}^{c}(z)$ $-\mathcal{G}_{n^{\prime}, n^{\prime}}^{v}(z)$. Thus, the monotonically increasing component of the two $\mathcal{G}$ functions due to the static carrier-induced strain

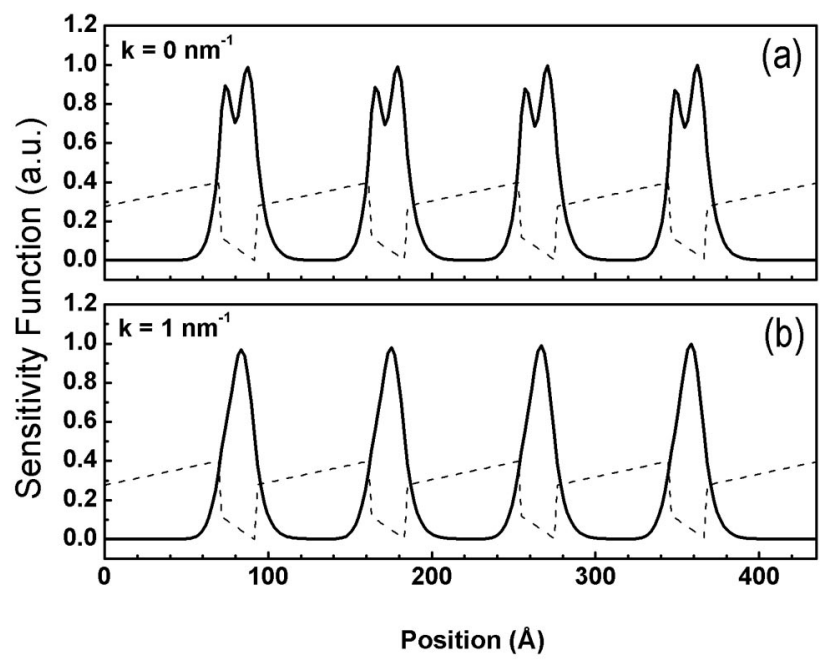

FIG. 4. The normalized sensitivity function related to $\mathrm{HH} 2$ and $\mathrm{C} 1$ at transverse wave vector (a) $k_{t}=0$ and (b) $k_{t}=1 \mathrm{~nm}^{-1}$. 


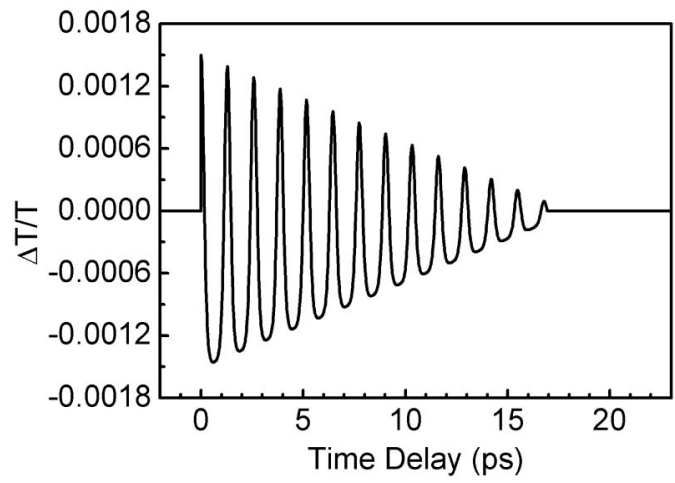

FIG. 5. Calculated transient probe transmission change as a function of time delay. We have subtracted the contribution from the static carrier-induced strain after the coherent waves leave the MQW.

cancels each other, resulting in a quasiperiodic dependence of $\mathcal{F}$ on $z$. Figure 3 is the sensitivity function for optical transition from $\mathrm{C} 1$ to $\mathrm{HH} 1$ subbands, and the case for $\mathrm{C} 1-$ $\mathrm{HH} 2$ optical transition is shown in Fig. 4. The dashed line indicates the conduction band edge of MQW. As can be seen from the figures, the sensitivity functions assume a nonzero value, mainly within the $\mathrm{QW}$ region. This means that when the strain pulse travels into the wells, the optical transmission changes significantly.

Figures 3(a) and 4(a) show the sensitivity functions with resonant in-plane wave vector $k_{0}=0$ for $\mathrm{C} 1-\mathrm{HH} 1$ and $\mathrm{C} 1-$ $\mathrm{HH} 2$ transitions, respectively. The results for corresponding transitions taking place at $k_{0}=1 \mathrm{~nm}^{-1}$ are shown in Figs. 3(b) and 4(b), respectively. As can be seen from Fig. 3, the wave form of $\mathrm{C} 1-\mathrm{HH} 1$ sensitivity function depends only weakly on the in-plane wave vector $k_{0}$. However, the wave form of $\mathrm{C} 1-\mathrm{HH} 2$ sensitivity function for transition at $k_{0}$ $=0$ differs significantly from that at $k_{0}=1 \mathrm{~nm}^{-1}$. The reason is as follows. For transitions at the band edge, the main contribution to the hole self-energy function $\mathcal{G}_{n^{\prime}, n^{\prime}}^{v}(z)$ comes from the heavy-hole component, whose wave function has a node within each QW. This results in the two peaks of the sensitivity function, as shown in Fig. 4(a). However, for optical transitions away from the band edge, contributions from other hole components due to valence-band mixing smooth the hole wave function. As a result, the resulting sensitivity function is similar to that of $\mathrm{C} 1-\mathrm{HH} 1$ transition.

We now combine the obtained time-varying strain field in Sec. II and the sensitivity function in Sec. IV to calculate the transient probe transmission change $\Delta T / T(t)$. The result is shown in Fig. 5. In the calculation, we assume a 14-period InGaN MQW. We also subtract the background contribution from the static carrier-induced strain field, which resides in the MQW after radiating components leave the QWs. From the figure, there is a total of 14 peaks with a linearly decaying envelope. Each peak corresponds to a maximum overlap of the sensitivity function with the strain field of the acoustic pulse trains. As the two trains propagate outward, the magnitude of maximum overlap decreases linearly.

The experimentally measured trace obtained by transient pump-probe measurement ${ }^{13}$ on a 14-period $22-\AA / 70-\AA$ $\mathrm{In}_{0.1} \mathrm{Ga}_{0.9} \mathrm{~N} / \mathrm{GaN}$ MQWs is shown in Fig. 6 for comparison

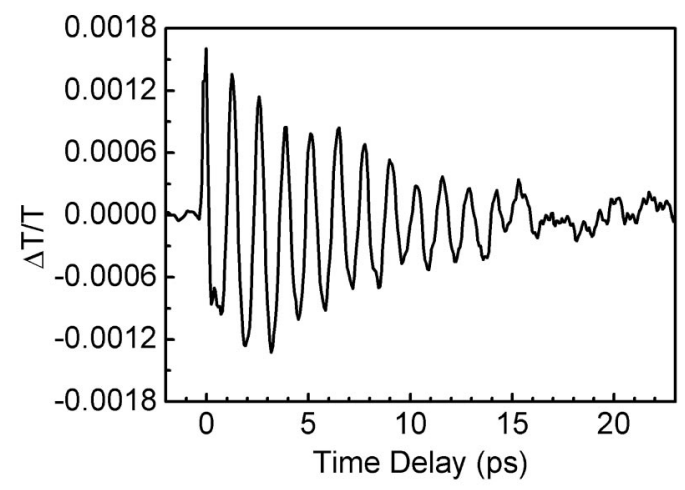

FIG. 6. Measured transient probe transmission change without background.

with the theoretical result. The MQW was grown on a 2.2 $\mu \mathrm{m}$ Si-doped GaN thin film and capped with a $0.1 \mu \mathrm{m}$ $\mathrm{Al}_{0.1} \mathrm{Ga}_{0.9} \mathrm{~N}$ layer. The pump-probe photon energy was 3.17 $\mathrm{eV}(390 \mathrm{~nm})$. Incident pump power was $28 \mathrm{~mW}$, with a corresponding optical fluence of $3.6 \times 10^{-4} \mathrm{~J} / \mathrm{cm}^{2}$. By measuring the reflected and transmitted power before and after the sample, the pump-induced two-dimensional carrier density in a well was estimated to be $8 \times 10^{12} \mathrm{~cm}^{-2}$, with a measured focal beam diameter of $14 \mu \mathrm{m}$. The experimental trace shows a linearly decaying envelope with 14 peaks. The agreement with theoretical result is fairly good.

\section{CONCLUSION}

In summary, we have presented a simple continuum theory for the photogeneration of coherent LA phonons in wurtzite InGaN/GaN MQWs. The theory is based on macroscopic constitutive relations of piezoelectric semiconductors, continuum elastic equations, and Poisson equation. Neglecting the small difference of elastic properties between QWs and barriers, the lattice displacement of a MQW obeys a loaded-string equation, as proposed previously. ${ }^{17}$ We solve the loaded-string equation to obtain the time-varying strain field of coherent LA phonons. The results are combined with sensitivity functions to calculate the transient optical transmission changes.

Starting from the microscopic LA-phonon-electron interaction Hamiltonian, we obtain analytic expressions for optical absorptions of MQW modulated by coherent acoustic phonons. Similar to its optical counterpart, the laser, CAPs can be regarded as a semiclassical wave. The macroscopic acoustic wave introduces a renormalization to the singleparticle energy and also results in couplings between carriers in different subbands. The interband density matrix elements are solved under the assumption that the electron system adiabatically follows the motion of CAPs. Optical absorption modulation results from the phonon-induced shift of the resonant transition point.

By expressing the phonon-induced renormalization using the strain field, we can write the probe transmission change as an overlapping integral of strain with a sensitivity function. We also derive analytic expressions for the sensitivity function. Dependences of the sensitivity function on optical transition point for different subband transitions are 
also studied. Based on these results, we calculate the transient probe transmission change and compare the results with the experimentally measured trace. A fairly good agreement is obtained.

\section{ACKNOWLEDGMENTS}

The authors would like to acknowledge the stimulating scientific discussions with C. J. Stanton. The InGaN MQW sample was kindly provided by S. P. DenBaars and S. Kellers. This work is sponsored by National Science Council of Taiwan under project number NSC 92-2112-M-002-044 and NSC 92-2120-M-002-004. One of the authors (K.-H.L.) would like to thank SiS Education Foundation for financial support.

${ }^{1}$ F. Alsina, P. V. Santos, H.-P. Schonherr, W. Seidel, K. H. Ploog, and R. Notzel, Phys. Rev. B 66, 165330 (2002).

${ }^{2}$ F. Alsina, P. V. Santos, H.-P. Schonherr, R. Notzel, and K. H. Ploog, Phys. Rev. B 67, 161305 (2003).

${ }^{3}$ C. Rocke, S. Zimmermann, A. Wixforth, J. P. Kotthaus, G. Bohm, and G. Weimann, Phys. Rev. Lett. 78, 4099 (1997).

${ }^{4}$ C. Thomsen et al., Phys. Rev. Lett. 53, 989 (1984).

${ }^{5}$ K. A. Nelson, D. R. Lutz, M. D. Fayer, and L. Madison, Phys. Rev. B 24, 3261 (1981).

${ }^{6}$ Y.-K. Huang et al., Appl. Phys. Lett. 79, 3361 (2001).
${ }^{7}$ M. D. Cummings and A. Y. Elezzabi, Appl. Phys. Lett. 79, 770 (2001).

${ }^{8}$ A. Yamamoto, T. Mishina, Y. Masumoto, and M. Nakayama, Phys. Rev. Lett. 73, 740 (1994).

${ }^{9}$ A. Bartels, T. Dekorsy, H. Kurz, and K. Köhler, Phys. Rev. Lett. 82, 1044 (1999).

${ }^{10}$ T. D. Krauss and F. W. Wise, Phys. Rev. Lett. 79, 5102 (1997).

${ }^{11}$ M. Nisoli et al., Phys. Rev. B 55, R13424 (1997).

${ }^{12}$ C.-K. Sun et al., Appl. Phys. Lett. 75, 1249 (1999).

${ }^{13}$ C.-K. Sun J.-C. Liang, and X.-Y. Yu, Phys. Rev. Lett. 84, 179 (2000).

${ }^{14}$ C.-K. Sun et al., Appl. Phys. Lett. 78, 1201 (2001).

${ }^{15}$ Ü. Özgür, C.-W. Lee, and H. O. Everitt, Phys. Rev. Lett. 86, 5604 (2001).

${ }^{16}$ G.-W. Chern, K.-H. Lin, Y.-K. Huang, and C.-K. Sun, Phys. Rev. B 67, 121303 (2003).

${ }^{17}$ G. D. Sanders, C. J. Stanton, and C. S. Kim, Phys. Rev. B 64, 235316 (2001).

${ }^{18}$ S. L. Chuang, Physics of Optoelectronic Devices (Wiley, New York, 1995).

${ }^{19}$ D. A. B. Miller, D. S. Chemla, and S. Schmitt-Rink, Phys. Rev. B 33, 6976 (1986).

${ }^{20}$ C. Thomsen, H. T. Grahn, H. J. Maris, and J. Tauc, Phys. Rev. B 34, 4129 (1986).

${ }^{21}$ G.-W. Chern and C.-K. Sun (unpublished).

${ }^{22}$ W. Schäfer and M. Wegener, Semiconductor Optics and Transport Phenomena (Springer, Berlin, 2002).

${ }^{23} \mathrm{H}$. Haug and S. W. Koch, Quantum Theory of the Optical and Electronic Properties of Semiconductors (World Scientific, Singapore, 1993).

${ }^{24}$ G. D. Sanders, C. J. Stanton, and C. S. Kim, Phys. Rev. B 66, 079903(E) (2002). 